\title{
Erratum: Search for direct pair production of scalar top quarks in the single- and dilepton channels in proton-proton collisions at $\sqrt{s}=8 \mathrm{TeV}$
}

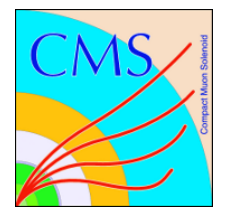

\section{The CMS collaboration}

E-mail: cms-publication-committee-chair@cern.ch

ERRATUM TO: JHEP07(2016)027

ArXiv EPrint: 1602.03169 
In the original paper, the wrong plot was used in figure 4. The corrected figure is given below.
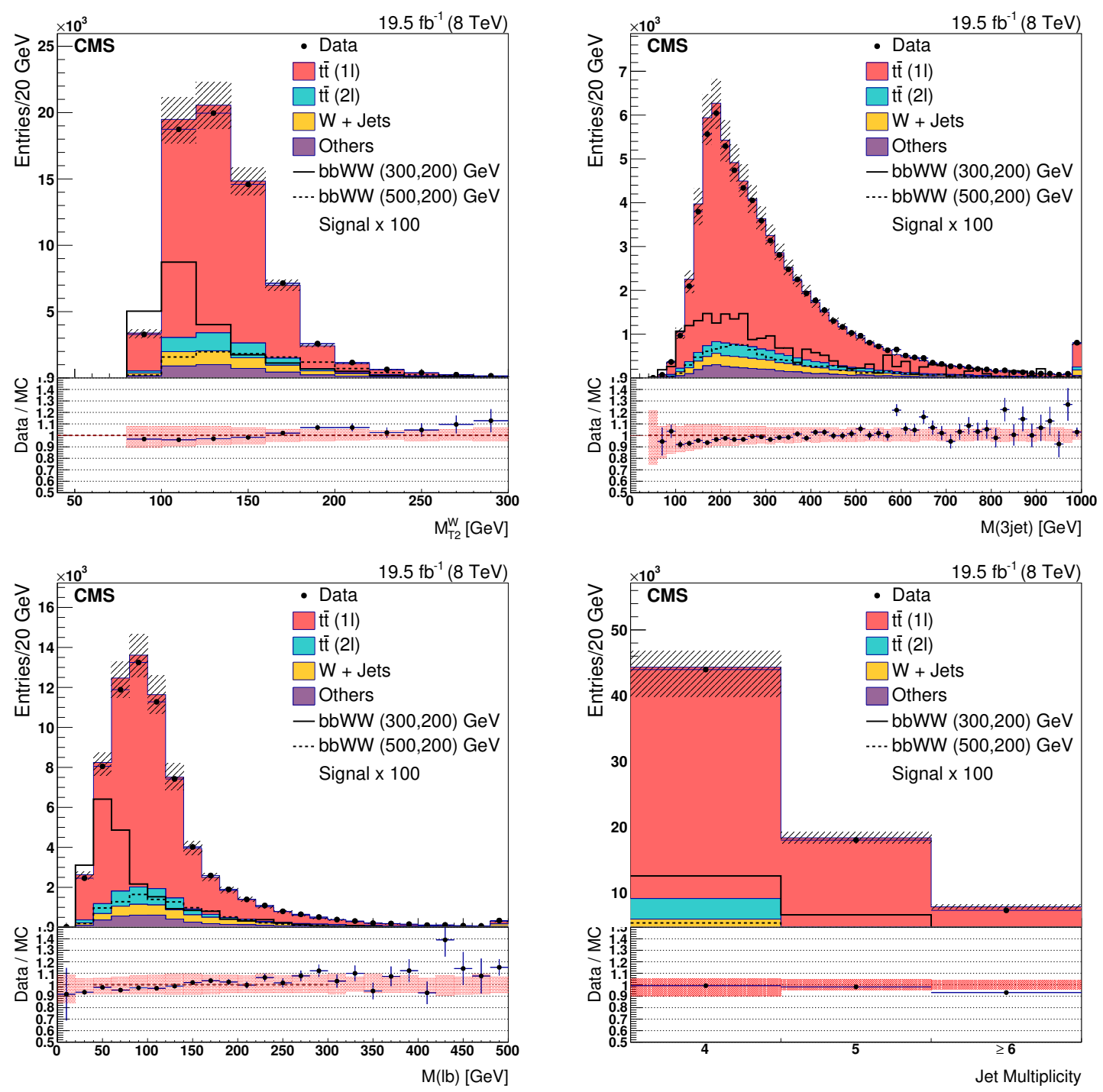

Figure 4. Distributions of different variables in both data and simulation, for both e and $\mu$ final states at the preselection level without the $M_{\mathrm{T}}$ requirement. From left to right: $M_{\mathrm{T} 2}^{\mathrm{W}}, M(3$ jet$)$, $M(\ell \mathrm{b})$ and $N$ (jets). The hatched region represents the quadratic sum of statistical and JES simulation uncertainties. The lower panel shows the ratio of data to total simulation background, with the red band representing the uncertainties mentioned in the text. Two signal mass points of the bbWW decay mode $(x=0.75)$ are represented by open histograms, dashed and solid, with their cross sections scaled by 100 ; the two mass points $\left(m\left(\widetilde{\mathrm{t}}_{1}\right), m\left(\widetilde{\chi}_{1}^{0}\right)\right)$ are $(300,200)$ and $(500,200) \mathrm{GeV}$.

Open Access. This article is distributed under the terms of the Creative Commons Attribution License (CC-BY 4.0), which permits any use, distribution and reproduction in any medium, provided the original author(s) and source are credited. 


\section{The CMS collaboration}

\section{Yerevan Physics Institute, Yerevan, Armenia}

V. Khachatryan, A.M. Sirunyan, A. Tumasyan

Institut für Hochenergiephysik der OeAW, Wien, Austria

W. Adam, E. Asilar, T. Bergauer, J. Brandstetter, E. Brondolin, M. Dragicevic, J. Erö, M. Flechl, M. Friedl, R. Frühwirth'1 ${ }^{1}$, V.M. Ghete, C. Hartl, N. Hörmann, J. Hrubec, M. Jeitler ${ }^{1}$, V. Knünz, A. König, M. Krammer ${ }^{1}$, I. Krätschmer, D. Liko, T. Matsushita, I. Mikulec, D. Rabady ${ }^{2}$, N. Rad, B. Rahbaran, H. Rohringer, J. Schieck ${ }^{1}$, R. Schöfbeck, J. Strauss, W. Treberer-Treberspurg, W. Waltenberger, C.-E. Wulz ${ }^{1}$

National Centre for Particle and High Energy Physics, Minsk, Belarus

V. Mossolov, N. Shumeiko, J. Suarez Gonzalez

Universiteit Antwerpen, Antwerpen, Belgium

S. Alderweireldt, T. Cornelis, E.A. De Wolf, X. Janssen, A. Knutsson, J. Lauwers, S. Luyckx, M. Van De Klundert, H. Van Haevermaet, P. Van Mechelen, N. Van Remortel, A. Van Spilbeeck

\section{Vrije Universiteit Brussel, Brussel, Belgium}

S. Abu Zeid, F. Blekman, J. D'Hondt, N. Daci, I. De Bruyn, K. Deroover, N. Heracleous, J. Keaveney, S. Lowette, L. Moreels, A. Olbrechts, Q. Python, D. Strom, S. Tavernier, W. Van Doninck, P. Van Mulders, G.P. Van Onsem, I. Van Parijs

\section{Université Libre de Bruxelles, Bruxelles, Belgium}

P. Barria, H. Brun, C. Caillol, B. Clerbaux, G. De Lentdecker, W. Fang, G. Fasanella, L. Favart, R. Goldouzian, A. Grebenyuk, G. Karapostoli, T. Lenzi, A. Léonard, T. Maerschalk, A. Marinov, L. Perniè, A. Randle-conde, T. Seva, C. Vander Velde, P. Vanlaer, R. Yonamine, F. Zenoni, F. Zhang ${ }^{3}$

\section{Ghent University, Ghent, Belgium}

K. Beernaert, L. Benucci, A. Cimmino, S. Crucy, D. Dobur, A. Fagot, G. Garcia, M. Gul, J. Mccartin, A.A. Ocampo Rios, D. Poyraz, D. Ryckbosch, S. Salva, M. Sigamani, M. Tytgat, W. Van Driessche, E. Yazgan, N. Zaganidis

\section{Université Catholique de Louvain, Louvain-la-Neuve, Belgium}

S. Basegmez, C. Beluffi ${ }^{4}$, O. Bondu, S. Brochet, G. Bruno, A. Caudron, L. Ceard, C. Delaere, D. Favart, L. Forthomme, A. Giammanco ${ }^{5}$, A. Jafari, P. Jez, M. Komm, V. Lemaitre, A. Mertens, M. Musich, C. Nuttens, L. Perrini, K. Piotrzkowski, A. Popov ${ }^{6}$, L. Quertenmont, M. Selvaggi, M. Vidal Marono

\section{Université de Mons, Mons, Belgium}

N. Beliy, G.H. Hammad

\section{Centro Brasileiro de Pesquisas Fisicas, Rio de Janeiro, Brazil}

W.L. Aldá Júnior, F.L. Alves, G.A. Alves, L. Brito, M. Correa Martins Junior, M. Hamer, C. Hensel, A. Moraes, M.E. Pol, P. Rebello Teles 
Universidade do Estado do Rio de Janeiro, Rio de Janeiro, Brazil

E. Belchior Batista Das Chagas, W. Carvalho, J. Chinellato ${ }^{7}$, A. Custódio, E.M. Da Costa, D. De Jesus Damiao, C. De Oliveira Martins, S. Fonseca De Souza, L.M. Huertas Guativa, H. Malbouisson, D. Matos Figueiredo, C. Mora Herrera, L. Mundim, H. Nogima, W.L. Prado Da Silva, A. Santoro, A. Sznajder, E.J. Tonelli Manganote ${ }^{7}$, A. Vilela Pereira

Universidade Estadual Paulista $^{a}$, Universidade Federal do ABC ${ }^{b}$, São Paulo, Brazil

S. Ahuja ${ }^{a}$, C.A. Bernardes ${ }^{b}$, A. De Souza Santos ${ }^{b}$, S. Dogra ${ }^{a}$, T.R. Fernandez Perez Tomei ${ }^{a}$, E.M. Gregores ${ }^{b}$, P.G. Mercadante ${ }^{b}$, C.S. Moon ${ }^{a, 8}$, S.F. Novaes ${ }^{a}$, Sandra S. Padula ${ }^{a}$, D. Romero Abad, J.C. Ruiz Vargas

Institute for Nuclear Research and Nuclear Energy, Sofia, Bulgaria

A. Aleksandrov, R. Hadjiiska, P. Iaydjiev, M. Rodozov, S. Stoykova, G. Sultanov, M. Vutova

University of Sofia, Sofia, Bulgaria

A. Dimitrov, I. Glushkov, L. Litov, B. Pavlov, P. Petkov

Institute of High Energy Physics, Beijing, China

M. Ahmad, J.G. Bian, G.M. Chen, H.S. Chen, M. Chen, T. Cheng, R. Du, C.H. Jiang,

D. Leggat, R. Plestina ${ }^{9}$, F. Romeo, S.M. Shaheen, A. Spiezia, J. Tao, C. Wang, Z. Wang,

H. Zhang

State Key Laboratory of Nuclear Physics and Technology, Peking University, Beijing, China

C. Asawatangtrakuldee, Y. Ban, Q. Li, S. Liu, Y. Mao, S.J. Qian, D. Wang, Z. Xu

Universidad de Los Andes, Bogota, Colombia

C. Avila, A. Cabrera, L.F. Chaparro Sierra, C. Florez, J.P. Gomez, B. Gomez Moreno, J.C. Sanabria

University of Split, Faculty of Electrical Engineering, Mechanical Engineering and Naval Architecture, Split, Croatia

N. Godinovic, D. Lelas, I. Puljak, P.M. Ribeiro Cipriano

University of Split, Faculty of Science, Split, Croatia

Z. Antunovic, M. Kovac

Institute Rudjer Boskovic, Zagreb, Croatia

V. Brigljevic, K. Kadija, J. Luetic, S. Micanovic, L. Sudic

University of Cyprus, Nicosia, Cyprus

A. Attikis, G. Mavromanolakis, J. Mousa, C. Nicolaou, F. Ptochos, P.A. Razis, H. Rykaczewski

Charles University, Prague, Czech Republic

M. Bodlak, M. Finger ${ }^{10}$, M. Finger Jr. ${ }^{10}$ 
Academy of Scientific Research and Technology of the Arab Republic of Egypt, Egyptian Network of High Energy Physics, Cairo, Egypt

Y. Assran ${ }^{11,12}$, S. Elgammal ${ }^{11}$, A. Ellithi Kamel ${ }^{13,13}$, M.A. Mahmoud ${ }^{14,11}$

National Institute of Chemical Physics and Biophysics, Tallinn, Estonia

B. Calpas, M. Kadastik, M. Murumaa, M. Raidal, A. Tiko, C. Veelken

Department of Physics, University of Helsinki, Helsinki, Finland

P. Eerola, J. Pekkanen, M. Voutilainen

Helsinki Institute of Physics, Helsinki, Finland

J. Härkönen, V. Karimäki, R. Kinnunen, T. Lampén, K. Lassila-Perini, S. Lehti, T. Lindén, P. Luukka, T. Peltola, J. Tuominiemi, E. Tuovinen, L. Wendland

Lappeenranta University of Technology, Lappeenranta, Finland

J. Talvitie, T. Tuuva

DSM/IRFU, CEA/Saclay, Gif-sur-Yvette, France

M. Besancon, F. Couderc, M. Dejardin, D. Denegri, B. Fabbro, J.L. Faure, C. Favaro, F. Ferri, S. Ganjour, A. Givernaud, P. Gras, G. Hamel de Monchenault, P. Jarry, E. Locci, M. Machet, J. Malcles, J. Rander, A. Rosowsky, M. Titov, A. Zghiche

Laboratoire Leprince-Ringuet, Ecole Polytechnique, IN2P3-CNRS, Palaiseau, France

I. Antropov, S. Baffioni, F. Beaudette, P. Busson, L. Cadamuro, E. Chapon, C. Charlot, O. Davignon, N. Filipovic, R. Granier de Cassagnac, M. Jo, S. Lisniak, L. Mastrolorenzo, P. Miné, I.N. Naranjo, M. Nguyen, C. Ochando, G. Ortona, P. Paganini, P. Pigard, S. Regnard, R. Salerno, J.B. Sauvan, Y. Sirois, T. Strebler, Y. Yilmaz, A. Zabi

Institut Pluridisciplinaire Hubert Curien, Université de Strasbourg, Université de Haute Alsace Mulhouse, CNRS/IN2P3, Strasbourg, France

J.-L. Agram ${ }^{15}$, J. Andrea, A. Aubin, D. Bloch, J.-M. Brom, M. Buttignol, E.C. Chabert, N. Chanon, C. Collard, E. Conte ${ }^{15}$, X. Coubez, J.-C. Fontaine ${ }^{15}$, D. Gelé, U. Goerlach, C. Goetzmann, A.-C. Le Bihan, J.A. Merlin², K. Skovpen, P. Van Hove

Centre de Calcul de l'Institut National de Physique Nucleaire et de Physique des Particules, CNRS/IN2P3, Villeurbanne, France

S. Gadrat

Université de Lyon, Université Claude Bernard Lyon 1, CNRS-IN2P3, Institut de Physique Nucléaire de Lyon, Villeurbanne, France

S. Beauceron, C. Bernet, G. Boudoul, E. Bouvier, C.A. Carrillo Montoya, R. Chierici, D. Contardo, B. Courbon, P. Depasse, H. El Mamouni, J. Fan, J. Fay, S. Gascon, M. Gouzevitch, B. Ille, F. Lagarde, I.B. Laktineh, M. Lethuillier, L. Mirabito, A.L. Pequegnot, S. Perries, J.D. Ruiz Alvarez, D. Sabes, L. Sgandurra, V. Sordini, M. Vander Donckt, P. Verdier, S. Viret

Georgian Technical University, Tbilisi, Georgia

T. Toriashvili ${ }^{16}$ 
Tbilisi State University, Tbilisi, Georgia

Z. Tsamalaidze ${ }^{10}$

\section{RWTH Aachen University, I. Physikalisches Institut, Aachen, Germany}

C. Autermann, S. Beranek, L. Feld, A. Heister, M.K. Kiesel, K. Klein, M. Lipinski,

A. Ostapchuk, M. Preuten, F. Raupach, S. Schael, J.F. Schulte, T. Verlage, H. Weber, V. Zhukov ${ }^{6}$

RWTH Aachen University, III. Physikalisches Institut A, Aachen, Germany M. Ata, M. Brodski, E. Dietz-Laursonn, D. Duchardt, M. Endres, M. Erdmann, S. Erdweg, T. Esch, R. Fischer, A. Güth, T. Hebbeker, C. Heidemann, K. Hoepfner, S. Knutzen, P. Kreuzer, M. Merschmeyer, A. Meyer, P. Millet, S. Mukherjee, M. Olschewski, K. Padeken, P. Papacz, T. Pook, M. Radziej, H. Reithler, M. Rieger, F. Scheuch, L. Sonnenschein, D. Teyssier, S. Thüer

\section{RWTH Aachen University, III. Physikalisches Institut B, Aachen, Germany}

V. Cherepanov, Y. Erdogan, G. Flügge, H. Geenen, M. Geisler, F. Hoehle, B. Kargoll, T. Kress, A. Künsken, J. Lingemann, A. Nehrkorn, A. Nowack, I.M. Nugent, C. Pistone, O. Pooth, A. Stahl

\section{Deutsches Elektronen-Synchrotron, Hamburg, Germany}

M. Aldaya Martin, I. Asin, N. Bartosik, O. Behnke, U. Behrens, K. Borras ${ }^{17}$, A. Burgmeier, A. Campbell, C. Contreras-Campana, F. Costanza, C. Diez Pardos, G. Dolinska, S. Dooling, T. Dorland, G. Eckerlin, D. Eckstein, T. Eichhorn, G. Flucke, E. Gallo ${ }^{18}$, J. Garay Garcia, A. Geiser, A. Gizhko, P. Gunnellini, J. Hauk, M. Hempel ${ }^{19}$, H. Jung, A. Kalogeropoulos, O. Karacheban ${ }^{19}$, M. Kasemann, P. Katsas, J. Kieseler, C. Kleinwort, I. Korol, D. Krücker, W. Lange, J. Leonard, K. Lipka, A. Lobanov, W. Lohmann ${ }^{19}$, R. Mankel, I.-A. Melzer-Pellmann, A.B. Meyer, G. Mittag, J. Mnich, A. Mussgiller, S. NaumannEmme, A. Nayak, E. Ntomari, H. Perrey, D. Pitzl, R. Placakyte, A. Raspereza, B. Roland, M.Ö. Sahin, P. Saxena, T. Schoerner-Sadenius, C. Seitz, S. Spannagel, N. Stefaniuk, K.D. Trippkewitz, R. Walsh, C. Wissing

\section{University of Hamburg, Hamburg, Germany}

V. Blobel, M. Centis Vignali, A.R. Draeger, J. Erfle, E. Garutti, K. Goebel, D. Gonzalez, M. Görner, J. Haller, M. Hoffmann, R.S. Höing, A. Junkes, R. Klanner, R. Kogler, N. Kovalchuk, T. Lapsien, T. Lenz, I. Marchesini, D. Marconi, M. Meyer, D. Nowatschin, J. Ott, F. Pantaleo ${ }^{2}$, T. Peiffer, A. Perieanu, N. Pietsch, J. Poehlsen, D. Rathjens, C. Sander, C. Scharf, P. Schleper, E. Schlieckau, A. Schmidt, S. Schumann, J. Schwandt, V. Sola, H. Stadie, G. Steinbrück, F.M. Stober, H. Tholen, D. Troendle, E. Usai, L. Vanelderen, A. Vanhoefer, B. Vormwald

\section{Institut für Experimentelle Kernphysik, Karlsruhe, Germany}

C. Barth, C. Baus, J. Berger, C. Böser, E. Butz, T. Chwalek, F. Colombo, W. De Boer, A. Descroix, A. Dierlamm, S. Fink, F. Frensch, R. Friese, M. Giffels, A. Gilbert, D. Haitz, F. Hartmann ${ }^{2}$, S.M. Heindl, U. Husemann, I. Katkov ${ }^{6}$, A. Kornmayer ${ }^{2}$, P. Lobelle Pardo, B. Maier, H. Mildner, M.U. Mozer, T. Müller, Th. Müller, M. Plagge, G. Quast, 
K. Rabbertz, S. Röcker, F. Roscher, M. Schröder, G. Sieber, H.J. Simonis, R. Ulrich, J. Wagner-Kuhr, S. Wayand, M. Weber, T. Weiler, S. Williamson, C. Wöhrmann, R. Wolf

Institute of Nuclear and Particle Physics (INPP), NCSR Demokritos, Aghia Paraskevi, Greece

G. Anagnostou, G. Daskalakis, T. Geralis, V.A. Giakoumopoulou, A. Kyriakis, D. Loukas,

A. Psallidas, I. Topsis-Giotis

National and Kapodistrian University of Athens, Athens, Greece

A. Agapitos, S. Kesisoglou, A. Panagiotou, N. Saoulidou, E. Tziaferi

University of Ioánnina, Ioánnina, Greece

I. Evangelou, G. Flouris, C. Foudas, P. Kokkas, N. Loukas, N. Manthos, I. Papadopoulos,

E. Paradas, J. Strologas

Wigner Research Centre for Physics, Budapest, Hungary

G. Bencze, C. Hajdu, A. Hazi, P. Hidas, D. Horvath ${ }^{20}$, F. Sikler, V. Veszpremi,

G. Vesztergombi ${ }^{21}$, A.J. Zsigmond

Institute of Nuclear Research ATOMKI, Debrecen, Hungary

N. Beni, S. Czellar, J. Karancsi ${ }^{22}$, J. Molnar, Z. Szillasi ${ }^{2}$

University of Debrecen, Debrecen, Hungary

M. Bartók ${ }^{23}$, A. Makovec, P. Raics, Z.L. Trocsanyi, B. Ujvari

National Institute of Science Education and Research, Bhubaneswar, India

S. Choudhury ${ }^{24}$, P. Mal, K. Mandal, D.K. Sahoo, N. Sahoo, S.K. Swain

Panjab University, Chandigarh, India

S. Bansal, S.B. Beri, V. Bhatnagar, R. Chawla, R. Gupta, U.Bhawandeep, A.K. Kalsi,

A. Kaur, M. Kaur, R. Kumar, A. Mehta, M. Mittal, J.B. Singh, G. Walia

University of Delhi, Delhi, India

Ashok Kumar, A. Bhardwaj, B.C. Choudhary, R.B. Garg, S. Malhotra, M. Naimuddin, N. Nishu, K. Ranjan, R. Sharma, V. Sharma

Saha Institute of Nuclear Physics, Kolkata, India

S. Bhattacharya, K. Chatterjee, S. Dey, S. Dutta, N. Majumdar, A. Modak, K. Mondal, S. Mukhopadhyay, A. Roy, D. Roy, S. Roy Chowdhury, S. Sarkar, M. Sharan

Bhabha Atomic Research Centre, Mumbai, India

A. Abdulsalam, R. Chudasama, D. Dutta, V. Jha, V. Kumar, A.K. Mohanty ${ }^{2}$, L.M. Pant, P. Shukla, A. Topkar

Tata Institute of Fundamental Research, Mumbai, India

T. Aziz, S. Banerjee, S. Bhowmik ${ }^{25}$, R.M. Chatterjee, R.K. Dewanjee, S. Dugad, S. Ganguly, S. Ghosh, M. Guchait, A. Gurtu ${ }^{26}$, Sa. Jain, G. Kole, S. Kumar, B. Mahakud, M. Maity ${ }^{25}$, G. Majumder, K. Mazumdar, S. Mitra, G.B. Mohanty, B. Parida, T. Sarkar ${ }^{25}$, N. Sur, B. Sutar, N. Wickramage ${ }^{27}$ 
Indian Institute of Science Education and Research (IISER), Pune, India

S. Chauhan, S. Dube, A. Kapoor, K. Kothekar, S. Sharma

Institute for Research in Fundamental Sciences (IPM), Tehran, Iran

H. Bakhshiansohi, H. Behnamian, S.M. Etesami ${ }^{28}$, A. Fahim ${ }^{29}$, M. Khakzad, M. Mohammadi Najafabadi, M. Naseri, S. Paktinat Mehdiabadi, F. Rezaei Hosseinabadi, B. Safarzadeh ${ }^{30}$, M. Zeinali

University College Dublin, Dublin, Ireland

M. Felcini, M. Grunewald

INFN Sezione di Bari ${ }^{a}$, Università di Bari ${ }^{b}$, Politecnico di Bari ${ }^{c}$, Bari, Italy

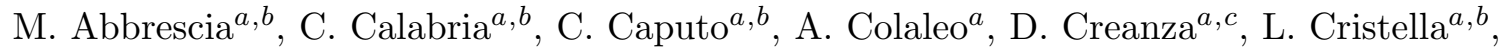
N. De Filippis ${ }^{a, c}$, M. De Palma ${ }^{a, b}$, L. Fiore ${ }^{a}$, G. Iaselli ${ }^{a, c}$, G. Maggi ${ }^{a, c}$, M. Maggi ${ }^{a}$, G. Miniello ${ }^{a, b}$, S. My ${ }^{a, c}$, S. Nuzzo ${ }^{a, b}$, A. Pompili ${ }^{a, b}$, G. Pugliese ${ }^{a, c}$, R. Radogna ${ }^{a, b}$, A. Ranieri ${ }^{a}$, G. Selvaggi ${ }^{a, b}$, L. Silvestris ${ }^{a, 2}$, R. Venditti ${ }^{a, b}$

INFN Sezione di Bologna ${ }^{a}$, Università di Bologna ${ }^{b}$, Bologna, Italy

G. Abbiendi ${ }^{a}$, C. Battilana ${ }^{2}$, D. Bonacorsi ${ }^{a, b}$, S. Braibant-Giacomelli ${ }^{a, b}$, L. Brigliadori $^{a, b}$, R. Campanini ${ }^{a, b}$, P. Capiluppi ${ }^{a, b}$, A. Castro ${ }^{a, b}$, F.R. Cavallo ${ }^{a}$, S.S. Chhibra ${ }^{a, b}$,

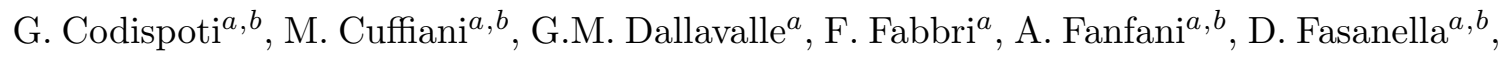
P. Giacomelli ${ }^{a}$, C. Grandi ${ }^{a}$, L. Guiducci ${ }^{a}, b$, S. Marcellini ${ }^{a}$, G. Masetti ${ }^{a}$, A. Montanari ${ }^{a}$,

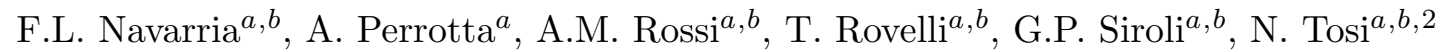

INFN Sezione di Catania ${ }^{a}$, Università di Catania ${ }^{b}$, Catania, Italy

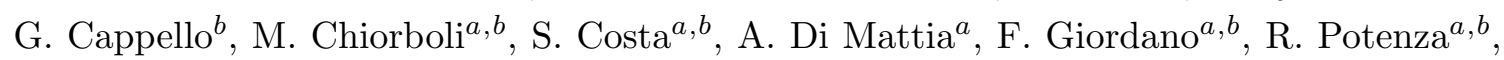
A. Tricomi ${ }^{a, b}$, C. Tuve ${ }^{a, b}$

INFN Sezione di Firenze ${ }^{a}$, Università di Firenze ${ }^{b}$, Firenze, Italy

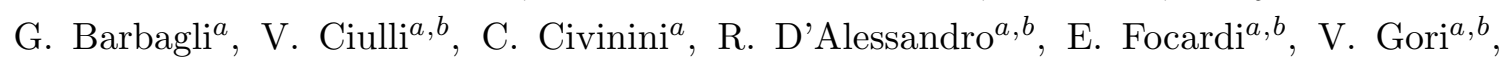
P. Lenzi ${ }^{a}, b$, M. Meschini ${ }^{a}$, S. Paoletti ${ }^{a}$, G. Sguazzoni ${ }^{a}$, L. Viliani ${ }^{a}, b, 2$

INFN Laboratori Nazionali di Frascati, Frascati, Italy

L. Benussi, S. Bianco, F. Fabbri, D. Piccolo, F. Primavera ${ }^{2}$

INFN Sezione di Genova ${ }^{a}$, Università di Genova ${ }^{b}$, Genova, Italy

V. Calvelli ${ }^{a, b}$, F. Ferro ${ }^{a}$, M. Lo Vetere ${ }^{a, b}$, M.R. Monge ${ }^{a, b}$, E. Robutti ${ }^{a}$, S. Tosi $^{a, b}$

INFN Sezione di Milano-Bicocca ${ }^{a}$, Università di Milano-Bicocca ${ }^{b}$, Milano, Italy

L. Brianza, M.E. Dinardo ${ }^{a, b}$, S. Fiorendi ${ }^{a}, b$, S. Gennai ${ }^{a}$, R. Gerosa ${ }^{a, b}$, A. Ghezzi $^{a, b}$, P. Govoni ${ }^{a, b}$, S. Malvezzi ${ }^{a}$, R.A. Manzoni ${ }^{a, b}, 2$, B. Marzocchi ${ }^{a, b}$, D. Menasce ${ }^{a}$, L. Moroni $^{a}$, M. Paganoni ${ }^{a, b}$, D. Pedrini ${ }^{a}$, S. Ragazzi ${ }^{a}, b$, N. Redaelli ${ }^{a}$, T. Tabarelli de Fatis ${ }^{a, b}$

INFN Sezione di Napoli ${ }^{a}$, Università di Napoli 'Federico II' ${ }^{b}$, Napoli, Italy, Università della Basilicata ${ }^{c}$, Potenza, Italy, Università G. Marconi ${ }^{d}$, Roma, Italy

S. Buontempo ${ }^{a}$, N. Cavallo $^{a, c}$, S. Di Guida ${ }^{a, d, 2}$, M. Esposito ${ }^{a, b}$, F. Fabozzi ${ }^{a, c}$,

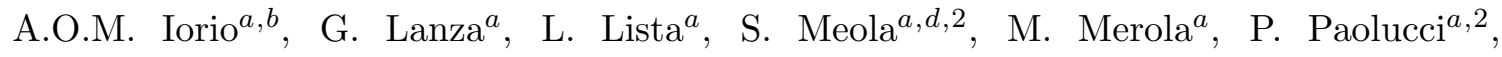
C. Sciacca ${ }^{a, b}$, F. Thyssen 
INFN Sezione di Padova ${ }^{a}$, Università di Padova ${ }^{b}$, Padova, Italy, Università di Trento ${ }^{c}$, Trento, Italy

P. Azzi ${ }^{a, 2}$, M. Bellato ${ }^{a}$, L. Benato ${ }^{a, b}$, D. Bisello ${ }^{a, b}$, A. Boletti $^{a}, b$, R. Carlin $^{a, b}$, P. Checchia $^{a}$,

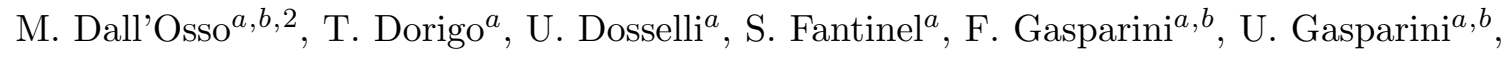

F. Gonella ${ }^{a}$, A. Gozzelino ${ }^{a}$, S. Lacaprara ${ }^{a}$, G. Maron $^{a, 31}$, F. Montecassiano $^{a}$, M. Passaseo $^{a}$, J. Pazzini ${ }^{a, b, 2}$, M. Pegoraro ${ }^{a}$, N. Pozzobon ${ }^{a, b}$, P. Ronchese ${ }^{a, b}$, M. Tosi ${ }^{a, b}$, S. Vanini ${ }^{a, b}$, S. Ventura ${ }^{a}$, M. Zanetti, A. Zucchetta ${ }^{a, b, 2}$, G. Zumerle Zu $^{a, b}$

INFN Sezione di Pavia ${ }^{a}$, Università di Pavia ${ }^{b}$, Pavia, Italy

A. Braghieri ${ }^{a}$, A. Magnani ${ }^{a, b}$, P. Montagna ${ }^{a, b}$, S.P. Ratti ${ }^{a, b}$, V. Re ${ }^{a}$, C. Riccardi ${ }^{a, b}$, P. Salvini ${ }^{a}$, I. Vai ${ }^{a, b}$, P. Vitulo ${ }^{a, b}$

INFN Sezione di Perugia ${ }^{a}$, Università di Perugia ${ }^{b}$, Perugia, Italy

L. Alunni Solestizi ${ }^{a, b}$, G.M. Bilei ${ }^{a}$, D. Ciangottini ${ }^{a, b, 2}$, L. Fanò ${ }^{a, b}$, P. Lariccia $^{a, b}$, G. Mantovani ${ }^{a, b}$, M. Menichelli ${ }^{a}$, A. Saha ${ }^{a}$, A. Santocchia ${ }^{a, b}$

INFN Sezione di Pisa ${ }^{a}$, Università di Pisa ${ }^{b}$, Scuola Normale Superiore di Pisa ${ }^{c}$, Pisa, Italy

K. Androsov ${ }^{a, 32}$, P. Azzurri ${ }^{a, 2}$, G. Bagliesi ${ }^{a}$, J. Bernardini ${ }^{a}$, T. Boccali ${ }^{a}$, R. Castaldi $^{a}$,

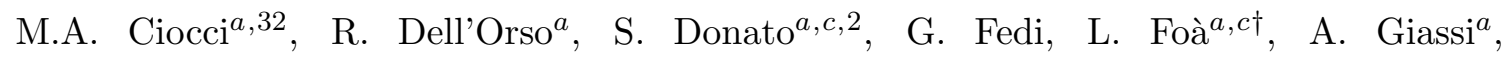

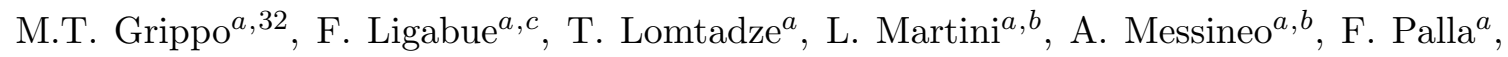

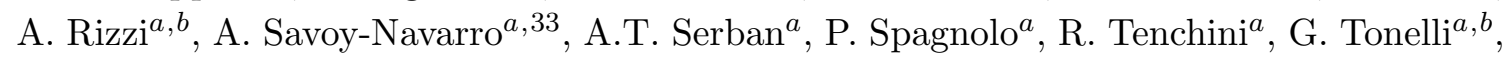
A. Venturi ${ }^{a}$, P.G. Verdini ${ }^{a}$

INFN Sezione di Roma ${ }^{a}$, Università di Roma ${ }^{b}$, Roma, Italy

L. Barone ${ }^{a, b}$, F. Cavallari ${ }^{a}$, G. D'imperio ${ }^{a, b, 2}$, D. Del Re ${ }^{a, b, 2}$, M. Diemoz ${ }^{a}$, S. Gelli ${ }^{a, b}$, C. Jorda ${ }^{a}$, E. Longo ${ }^{a, b}$, F. Margaroli ${ }^{a}, b$, P. Meridiani ${ }^{a}$, G. Organtini ${ }^{a, b}$, R. Paramatti ${ }^{a}$,

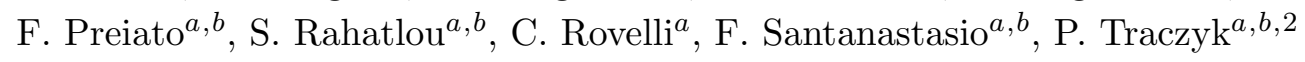

INFN Sezione di Torino ${ }^{a}$, Università di Torino ${ }^{b}$, Torino, Italy, Università del Piemonte Orientale ${ }^{c}$, Novara, Italy

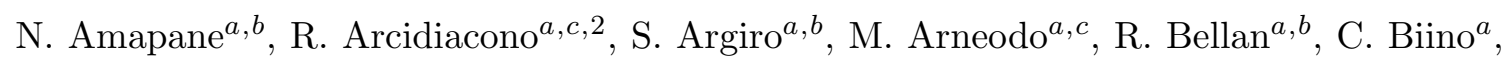

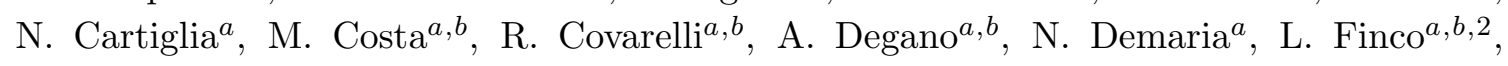

B. Kiani ${ }^{a, b}$, C. Mariotti ${ }^{a}$, S. Maselli ${ }^{a}$, E. Migliore ${ }^{a, b}$, V. Monaco ${ }^{a, b}$, E. Monteil ${ }^{a, b}$, M.M. Obertino $^{a, b}$, L. Pacher ${ }^{a, b}$, N. Pastrone ${ }^{a}$, M. Pelliccioni ${ }^{a}$, G.L. Pinna Angioni ${ }^{a}, b$, F. Ravera ${ }^{a, b}$, A. Romero ${ }^{a, b}$, M. Ruspa $^{a, c}$, R. Sacchi ${ }^{a, b}$, A. Solano $^{a, b}$, A. Staiano $^{a}$

INFN Sezione di Trieste ${ }^{a}$, Università di Trieste ${ }^{b}$, Trieste, Italy

S. Belforte ${ }^{a}$, V. Candelise ${ }^{a, b}$, M. Casarsa ${ }^{a}$, F. Cossutti ${ }^{a}$, G. Della Ricca ${ }^{a, b}$, B. Gobbo $^{a}$, C. La Licata ${ }^{a, b}$, M. Marone ${ }^{a, b}$, A. Schizzi ${ }^{a, b}$, A. Zanetti $^{a}$

Kangwon National University, Chunchon, Korea

A. Kropivnitskaya, S.K. Nam

Kyungpook National University, Daegu, Korea

D.H. Kim, G.N. Kim, M.S. Kim, D.J. Kong, S. Lee, Y.D. Oh, A. Sakharov, D.C. Son 
Chonbuk National University, Jeonju, Korea

J.A. Brochero Cifuentes, H. Kim, T.J. Kim

Chonnam National University, Institute for Universe and Elementary Particles, Kwangju, Korea

S. Song

Korea University, Seoul, Korea

S. Cho, S. Choi, Y. Go, D. Gyun, B. Hong, H. Kim, Y. Kim, B. Lee, K. Lee, K.S. Lee, S. Lee, J. Lim, S.K. Park, Y. Roh

Seoul National University, Seoul, Korea

H.D. Yoo

University of Seoul, Seoul, Korea

M. Choi, H. Kim, J.H. Kim, J.S.H. Lee, I.C. Park, G. Ryu, M.S. Ryu

Sungkyunkwan University, Suwon, Korea

Y. Choi, J. Goh, D. Kim, E. Kwon, J. Lee, I. Yu

Vilnius University, Vilnius, Lithuania

V. Dudenas, A. Juodagalvis, J. Vaitkus

National Centre for Particle Physics, Universiti Malaya, Kuala Lumpur, Malaysia

I. Ahmed, Z.A. Ibrahim, J.R. Komaragiri, M.A.B. Md Ali ${ }^{34}$, F. Mohamad Idris ${ }^{35}$, W.A.T. Wan Abdullah, M.N. Yusli, Z. Zolkapli

Centro de Investigacion y de Estudios Avanzados del IPN, Mexico City, Mexico

E. Casimiro Linares, H. Castilla-Valdez, E. De La Cruz-Burelo, I. Heredia-De La Cruz ${ }^{36}$,

A. Hernandez-Almada, R. Lopez-Fernandez, A. Sanchez-Hernandez

Universidad Iberoamericana, Mexico City, Mexico

S. Carrillo Moreno, F. Vazquez Valencia

Benemerita Universidad Autonoma de Puebla, Puebla, Mexico

I. Pedraza, H.A. Salazar Ibarguen, C. Uribe Estrada

Universidad Autónoma de San Luis Potosí, San Luis Potosí, Mexico

A. Morelos Pineda

University of Auckland, Auckland, New Zealand

D. Krofcheck

University of Canterbury, Christchurch, New Zealand

P.H. Butler

National Centre for Physics, Quaid-I-Azam University, Islamabad, Pakistan

A. Ahmad, M. Ahmad, Q. Hassan, H.R. Hoorani, W.A. Khan, T. Khurshid, M. Shoaib, M. Waqas 
National Centre for Nuclear Research, Swierk, Poland

H. Bialkowska, M. Bluj, B. Boimska, T. Frueboes, M. Górski, M. Kazana, K. Nawrocki, K. Romanowska-Rybinska, M. Szleper, P. Zalewski

Institute of Experimental Physics, Faculty of Physics, University of Warsaw, Warsaw, Poland

G. Brona, K. Bunkowski, A. Byszuk ${ }^{37}$, K. Doroba, A. Kalinowski, M. Konecki, J. Krolikowski, M. Misiura, M. Olszewski, M. Walczak

Laboratório de Instrumentação e Física Experimental de Partículas, Lisboa, Portugal

P. Bargassa, C. Beirão Da Cruz E Silva, A. Di Francesco, P. Faccioli, P.G. Ferreira Parracho, M. Gallinaro, J. Hollar, N. Leonardo, L. Lloret Iglesias, F. Nguyen, J. Rodrigues Antunes, J. Seixas, O. Toldaiev, D. Vadruccio, J. Varela, P. Vischia

Joint Institute for Nuclear Research, Dubna, Russia

S. Afanasiev, P. Bunin, M. Gavrilenko, I. Golutvin, I. Gorbunov, A. Kamenev, V. Karjavin, A. Lanev, A. Malakhov, V. Matveev ${ }^{38,39}$, P. Moisenz, V. Palichik, V. Perelygin, S. Shmatov, S. Shulha, N. Skatchkov, V. Smirnov, A. Zarubin

Petersburg Nuclear Physics Institute, Gatchina (St. Petersburg), Russia

V. Golovtsov, Y. Ivanov, V. Kim ${ }^{40}$, E. Kuznetsova, P. Levchenko, V. Murzin, V. Oreshkin, I. Smirnov, V. Sulimov, L. Uvarov, S. Vavilov, A. Vorobyev

Institute for Nuclear Research, Moscow, Russia

Yu. Andreev, A. Dermenev, S. Gninenko, N. Golubev, A. Karneyeu, M. Kirsanov, N. Krasnikov, A. Pashenkov, D. Tlisov, A. Toropin

Institute for Theoretical and Experimental Physics, Moscow, Russia

V. Epshteyn, V. Gavrilov, N. Lychkovskaya, V. Popov, I. Pozdnyakov, G. Safronov, A. Spiridonov, E. Vlasov, A. Zhokin

National Research Nuclear University 'Moscow Engineering Physics Institute' (MEPhI), Moscow, Russia

M. Chadeeva, R. Chistov, M. Danilov, V. Rusinov, E. Tarkovskii

P.N. Lebedev Physical Institute, Moscow, Russia

V. Andreev, M. Azarkin ${ }^{39}$, I. Dremin ${ }^{39}$, M. Kirakosyan, A. Leonidov ${ }^{39}$, G. Mesyats, S.V. Rusakov

Skobeltsyn Institute of Nuclear Physics, Lomonosov Moscow State University, Moscow, Russia

A. Baskakov, A. Belyaev, E. Boos, M. Dubinin ${ }^{41}$, L. Dudko, A. Ershov, A. Gribushin, V. Klyukhin, O. Kodolova, I. Lokhtin, I. Miagkov, S. Obraztsov, S. Petrushanko, V. Savrin, A. Snigirev 
State Research Center of Russian Federation, Institute for High Energy Physics, Protvino, Russia

I. Azhgirey, I. Bayshev, S. Bitioukov, V. Kachanov, A. Kalinin, D. Konstantinov, V. Krychkine, V. Petrov, R. Ryutin, A. Sobol, L. Tourtchanovitch, S. Troshin, N. Tyurin, A. Uzunian, A. Volkov

University of Belgrade, Faculty of Physics and Vinca Institute of Nuclear Sciences, Belgrade, Serbia

P. Adzic ${ }^{42}$, P. Cirkovic, D. Devetak, J. Milosevic, V. Rekovic

Centro de Investigaciones Energéticas Medioambientales y Tecnológicas (CIEMAT), Madrid, Spain

J. Alcaraz Maestre, E. Calvo, M. Cerrada, M. Chamizo Llatas, N. Colino, B. De La Cruz, A. Delgado Peris, A. Escalante Del Valle, C. Fernandez Bedoya, J.P. Fernández Ramos, J. Flix, M.C. Fouz, P. Garcia-Abia, O. Gonzalez Lopez, S. Goy Lopez, J.M. Hernandez, M.I. Josa, E. Navarro De Martino, A. Pérez-Calero Yzquierdo, J. Puerta Pelayo, A. Quintario Olmeda, I. Redondo, L. Romero, J. Santaolalla, M.S. Soares

Universidad Autónoma de Madrid, Madrid, Spain

C. Albajar, J.F. de Trocóniz, M. Missiroli, D. Moran

Universidad de Oviedo, Oviedo, Spain

J. Cuevas, J. Fernandez Menendez, S. Folgueras, I. Gonzalez Caballero, E. Palencia Cortezon, J.M. Vizan Garcia

Instituto de Física de Cantabria (IFCA), CSIC-Universidad de Cantabria, Santander, Spain

I.J. Cabrillo, A. Calderon, J.R. Castiñeiras De Saa, P. De Castro Manzano, M. Fernandez, J. Garcia-Ferrero, G. Gomez, A. Lopez Virto, J. Marco, R. Marco, C. Martinez Rivero, F. Matorras, J. Piedra Gomez, T. Rodrigo, A.Y. Rodríguez-Marrero, A. Ruiz-Jimeno, L. Scodellaro, N. Trevisani, I. Vila, R. Vilar Cortabitarte

CERN, European Organization for Nuclear Research, Geneva, Switzerland

D. Abbaneo, E. Auffray, G. Auzinger, M. Bachtis, P. Baillon, A.H. Ball, D. Barney, A. Benaglia, J. Bendavid, L. Benhabib, G.M. Berruti, P. Bloch, A. Bocci, A. Bonato, C. Botta, H. Breuker, T. Camporesi, R. Castello, G. Cerminara, M. D'Alfonso, D. d'Enterria, A. Dabrowski, V. Daponte, A. David, M. De Gruttola, F. De Guio, A. De Roeck, S. De Visscher, E. Di $\mathrm{Marco}^{43}$, M. Dobson, M. Dordevic, B. Dorney, T. du Pree, D. Duggan, M. Dünser, N. Dupont, A. Elliott-Peisert, G. Franzoni, J. Fulcher, W. Funk, D. Gigi, K. Gill, D. Giordano, M. Girone, F. Glege, R. Guida, S. Gundacker, M. Guthoff, J. Hammer, P. Harris, J. Hegeman, V. Innocente, P. Janot, H. Kirschenmann, M.J. Kortelainen, K. Kousouris, K. Krajczar, P. Lecoq, C. Lourenço, M.T. Lucchini, N. Magini, L. Malgeri, M. Mannelli, A. Martelli, L. Masetti, F. Meijers, S. Mersi, E. Meschi, F. Moortgat, S. Morovic, M. Mulders, M.V. Nemallapudi, H. Neugebauer, S. Orfanelli ${ }^{44}$, L. Orsini, L. Pape, E. Perez, M. Peruzzi, A. Petrilli, G. Petrucciani, A. Pfeiffer, M. Pierini, D. Piparo, A. Racz, T. Reis, G. Rolandi ${ }^{45}$, M. Rovere, M. Ruan, H. Sakulin, C. Schäfer, 
C. Schwick, M. Seidel, A. Sharma, P. Silva, M. Simon, P. Sphicas ${ }^{46}$, J. Steggemann, B. Stieger, M. Stoye, Y. Takahashi, D. Treille, A. Triossi, A. Tsirou, G.I. Veres ${ }^{21}$, N. Wardle, H.K. Wöhri, A. Zagozdzinska ${ }^{37}$, W.D. Zeuner

Paul Scherrer Institut, Villigen, Switzerland

W. Bertl, K. Deiters, W. Erdmann, R. Horisberger, Q. Ingram, H.C. Kaestli, D. Kotlinski, U. Langenegger, T. Rohe

Institute for Particle Physics, ETH Zurich, Zurich, Switzerland

F. Bachmair, L. Bäni, L. Bianchini, B. Casal, G. Dissertori, M. Dittmar, M. Donegà, P. Eller, C. Grab, C. Heidegger, D. Hits, J. Hoss, G. Kasieczka, P. Lecomte ${ }^{\dagger}$, W. Lustermann, B. Mangano, M. Marionneau, P. Martinez Ruiz del Arbol, M. Masciovecchio, M.T. Meinhard, D. Meister, F. Micheli, P. Musella, F. Nessi-Tedaldi, F. Pandolfi, J. Pata, F. Pauss, L. Perrozzi, M. Quittnat, M. Rossini, M. Schönenberger, A. Starodumov ${ }^{47}$, M. Takahashi, V.R. Tavolaro, K. Theofilatos, R. Wallny

Universität Zürich, Zurich, Switzerland

T.K. Aarrestad, C. Amsler ${ }^{48}$, L. Caminada, M.F. Canelli, V. Chiochia, A. De Cosa, C. Galloni, A. Hinzmann, T. Hreus, B. Kilminster, C. Lange, J. Ngadiuba, D. Pinna, G. Rauco, P. Robmann, D. Salerno, Y. Yang

\section{National Central University, Chung-Li, Taiwan}

M. Cardaci, K.H. Chen, T.H. Doan, Sh. Jain, R. Khurana, M. Konyushikhin, C.M. Kuo, W. Lin, Y.J. Lu, A. Pozdnyakov, S.S. Yu

National Taiwan University (NTU), Taipei, Taiwan

Arun Kumar, P. Chang, Y.H. Chang, Y.W. Chang, Y. Chao, K.F. Chen, P.H. Chen, C. Dietz, F. Fiori, U. Grundler, W.-S. Hou, Y. Hsiung, Y.F. Liu, R.-S. Lu, M. Miñano Moya, E. Petrakou, J.f. Tsai, Y.M. Tzeng

Chulalongkorn University, Faculty of Science, Department of Physics, Bangkok, Thailand

B. Asavapibhop, K. Kovitanggoon, G. Singh, N. Srimanobhas, N. Suwonjandee

Cukurova University, Adana, Turkey

A. Adiguzel, S. Cerci $^{49}$, S. Damarseckin, Z.S. Demiroglu, C. Dozen, I. Dumanoglu, F.H. Gecit, S. Girgis, G. Gokbulut, Y. Guler, E. Gurpinar, I. Hos, E.E. Kangal ${ }^{50}$, A. Kayis Topaksu, G. Onengut ${ }^{51}$, M. Ozcan, K. Ozdemir ${ }^{52}$, S. Ozturk ${ }^{53}$, A. Polatoz, B. Tali ${ }^{49}$, C. Zorbilmez

Middle East Technical University, Physics Department, Ankara, Turkey

B. Bilin, S. Bilmis, B. Isildak ${ }^{54}$, G. Karapinar ${ }^{55}$, M. Yalvac, M. Zeyrek

Bogazici University, Istanbul, Turkey

E. Gülmez, M. Kaya ${ }^{56}$, O. Kaya ${ }^{57}$, E.A. Yetkin ${ }^{58}$, T. Yetkin ${ }^{59}$

Istanbul Technical University, Istanbul, Turkey

A. Cakir, K. Cankocak, S. Sen ${ }^{60}$, F.I. Vardarlı 
Institute for Scintillation Materials of National Academy of Science of Ukraine, Kharkov, Ukraine

B. Grynyov

National Scientific Center, Kharkov Institute of Physics and Technology, Kharkov, Ukraine

L. Levchuk, P. Sorokin

University of Bristol, Bristol, United Kingdom

R. Aggleton, F. Ball, L. Beck, J.J. Brooke, E. Clement, D. Cussans, H. Flacher, J. Goldstein, M. Grimes, G.P. Heath, H.F. Heath, J. Jacob, L. Kreczko, C. Lucas, Z. Meng, D.M. Newbold ${ }^{61}$, S. Paramesvaran, A. Poll, T. Sakuma, S. Seif El Nasr-storey, S. Senkin, D. Smith, V.J. Smith

\section{Rutherford Appleton Laboratory, Didcot, United Kingdom}

K.W. Bell, A. Belyaev ${ }^{62}$, C. Brew, R.M. Brown, L. Calligaris, D. Cieri, D.J.A. Cockerill, J.A. Coughlan, K. Harder, S. Harper, E. Olaiya, D. Petyt, C.H. Shepherd-Themistocleous, A. Thea, I.R. Tomalin, T. Williams, S.D. Worm

\section{Imperial College, London, United Kingdom}

M. Baber, R. Bainbridge, O. Buchmuller, A. Bundock, D. Burton, S. Casasso, M. Citron, D. Colling, L. Corpe, P. Dauncey, G. Davies, A. De Wit, M. Della Negra, P. Dunne, A. Elwood, D. Futyan, G. Hall, G. Iles, R. Lane, R. Lucas ${ }^{61}$, L. Lyons, A.-M. Magnan, S. Malik, J. Nash, A. Nikitenko ${ }^{47}$, J. Pela, M. Pesaresi, D.M. Raymond, A. Richards, A. Rose, C. Seez, A. Tapper, K. Uchida, M. Vazquez Acosta ${ }^{63}$, T. Virdee, S.C. Zenz

Brunel University, Uxbridge, United Kingdom

J.E. Cole, P.R. Hobson, A. Khan, P. Kyberd, D. Leslie, I.D. Reid, P. Symonds, L. Teodorescu, M. Turner

Baylor University, Waco, USA

A. Borzou, K. Call, J. Dittmann, K. Hatakeyama, H. Liu, N. Pastika

The University of Alabama, Tuscaloosa, USA

O. Charaf, S.I. Cooper, C. Henderson, P. Rumerio

Boston University, Boston, USA

D. Arcaro, A. Avetisyan, T. Bose, D. Gastler, D. Rankin, C. Richardson, J. Rohlf, L. Sulak, D. Zou

\section{Brown University, Providence, USA}

J. Alimena, G. Benelli, E. Berry, D. Cutts, A. Ferapontov, A. Garabedian, J. Hakala, U. Heintz, O. Jesus, E. Laird, G. Landsberg, Z. Mao, M. Narain, S. Piperov, S. Sagir, R. Syarif

University of California, Davis, Davis, USA

R. Breedon, G. Breto, M. Calderon De La Barca Sanchez, S. Chauhan, M. Chertok, J. Conway, R. Conway, P.T. Cox, R. Erbacher, G. Funk, M. Gardner, W. Ko, R. Lander, 
C. Mclean, M. Mulhearn, D. Pellett, J. Pilot, F. Ricci-Tam, S. Shalhout, J. Smith, M. Squires, D. Stolp, M. Tripathi, S. Wilbur, R. Yohay

\section{University of California, Los Angeles, USA}

R. Cousins, P. Everaerts, A. Florent, J. Hauser, M. Ignatenko, D. Saltzberg, E. Takasugi, V. Valuev, M. Weber

University of California, Riverside, Riverside, USA

K. Burt, R. Clare, J. Ellison, J.W. Gary, G. Hanson, J. Heilman, M. Ivova PANEVA, P. Jandir, E. Kennedy, F. Lacroix, O.R. Long, M. Malberti, M. Olmedo Negrete, A. Shrinivas, H. Wei, S. Wimpenny, B. R. Yates

University of California, San Diego, La Jolla, USA

J.G. Branson, G.B. Cerati, S. Cittolin, R.T. D’Agnolo, M. Derdzinski, A. Holzner, R. Kelley, D. Klein, J. Letts, I. Macneill, D. Olivito, S. Padhi, M. Pieri, M. Sani, V. Sharma, S. Simon, M. Tadel, A. Vartak, S. Wasserbaech ${ }^{64}$, C. Welke, F. Würthwein, A. Yagil, G. Zevi Della Porta

\section{University of California, Santa Barbara, Santa Barbara, USA}

J. Bradmiller-Feld, C. Campagnari, A. Dishaw, V. Dutta, K. Flowers, M. Franco Sevilla, P. Geffert, C. George, F. Golf, L. Gouskos, J. Gran, J. Incandela, N. Mccoll, S.D. Mullin, J. Richman, D. Stuart, I. Suarez, C. West, J. Yoo

California Institute of Technology, Pasadena, USA

D. Anderson, A. Apresyan, A. Bornheim, J. Bunn, Y. Chen, J. Duarte, A. Mott, H.B. Newman, C. Pena, M. Spiropulu, J.R. Vlimant, S. Xie, R.Y. Zhu

\section{Carnegie Mellon University, Pittsburgh, USA}

M.B. Andrews, V. Azzolini, A. Calamba, B. Carlson, T. Ferguson, M. Paulini, J. Russ, M. Sun, H. Vogel, I. Vorobiev

\section{University of Colorado Boulder, Boulder, USA}

J.P. Cumalat, W.T. Ford, A. Gaz, F. Jensen, A. Johnson, M. Krohn, T. Mulholland, U. Nauenberg, K. Stenson, S.R. Wagner

\section{Cornell University, Ithaca, USA}

J. Alexander, A. Chatterjee, J. Chaves, J. Chu, S. Dittmer, N. Eggert, N. Mirman, G. Nicolas Kaufman, J.R. Patterson, A. Rinkevicius, A. Ryd, L. Skinnari, L. Soffi, W. Sun, S.M. Tan, W.D. Teo, J. Thom, J. Thompson, J. Tucker, Y. Weng, P. Wittich

\section{Fermi National Accelerator Laboratory, Batavia, USA}

S. Abdullin, M. Albrow, G. Apollinari, S. Banerjee, L.A.T. Bauerdick, A. Beretvas, J. Berryhill, P.C. Bhat, G. Bolla, K. Burkett, J.N. Butler, H.W.K. Cheung, F. Chlebana, S. Cihangir, V.D. Elvira, I. Fisk, J. Freeman, Z. Gecse, E. Gottschalk, L. Gray, D. Green, S. Grünendahl, O. Gutsche, J. Hanlon, D. Hare, R.M. Harris, S. Hasegawa, J. Hirschauer, Z. Hu, B. Jayatilaka, S. Jindariani, M. Johnson, U. Joshi, B. Klima, B. Kreis, S. Lammel, J. Lewis, J. Linacre, D. Lincoln, R. Lipton, T. Liu, R. Lopes De Sá, J. Lykken, K. Maeshima, J.M. Marraffino, S. Maruyama, D. Mason, P. McBride, P. Merkel, S. Mrenna, 
S. Nahn, C. Newman-Holmes ${ }^{\dagger}$, V. O’Dell, K. Pedro, O. Prokofyev, G. Rakness, E. SextonKennedy, A. Soha, W.J. Spalding, L. Spiegel, S. Stoynev, N. Strobbe, L. Taylor, S. Tkaczyk, N.V. Tran, L. Uplegger, E.W. Vaandering, C. Vernieri, M. Verzocchi, R. Vidal, M. Wang, H.A. Weber, A. Whitbeck

\section{University of Florida, Gainesville, USA}

D. Acosta, P. Avery, P. Bortignon, D. Bourilkov, A. Brinkerhoff, A. Carnes, M. Carver, D. Curry, S. Das, R.D. Field, I.K. Furic, J. Konigsberg, A. Korytov, K. Kotov, P. Ma, K. Matchev, H. Mei, P. Milenovic ${ }^{65}$, G. Mitselmakher, D. Rank, R. Rossin, L. Shchutska, M. Snowball, D. Sperka, N. Terentyev, L. Thomas, J. Wang, S. Wang, J. Yelton

\section{Florida International University, Miami, USA}

S. Hewamanage, S. Linn, P. Markowitz, G. Martinez, J.L. Rodriguez

\section{Florida State University, Tallahassee, USA}

A. Ackert, J.R. Adams, T. Adams, A. Askew, S. Bein, J. Bochenek, B. Diamond, J. Haas, S. Hagopian, V. Hagopian, K.F. Johnson, A. Khatiwada, H. Prosper, M. Weinberg

\section{Florida Institute of Technology, Melbourne, USA}

M.M. Baarmand, V. Bhopatkar, S. Colafranceschi ${ }^{66}$, M. Hohlmann, H. Kalakhety, D. Noonan, T. Roy, F. Yumiceva

\section{University of Illinois at Chicago (UIC), Chicago, USA}

M.R. Adams, L. Apanasevich, D. Berry, R.R. Betts, I. Bucinskaite, R. Cavanaugh, O. Evdokimov, L. Gauthier, C.E. Gerber, D.J. Hofman, P. Kurt, C. O'Brien, I.D. Sandoval Gonzalez, P. Turner, N. Varelas, Z. Wu, M. Zakaria, J. Zhang

\section{The University of Iowa, Iowa City, USA}

B. Bilki ${ }^{67}$, W. Clarida, K. Dilsiz, S. Durgut, R.P. Gandrajula, M. Haytmyradov, V. Khristenko, J.-P. Merlo, H. Mermerkaya ${ }^{68}$, A. Mestvirishvili, A. Moeller, J. Nachtman, H. Ogul, Y. Onel, F. Ozok ${ }^{69}$, A. Penzo, C. Snyder, E. Tiras, J. Wetzel, K. Yi

\section{Johns Hopkins University, Baltimore, USA}

I. Anderson, B.A. Barnett, B. Blumenfeld, N. Eminizer, D. Fehling, L. Feng, A.V. Gritsan, P. Maksimovic, M. Osherson, J. Roskes, A. Sady, U. Sarica, M. Swartz, M. Xiao, Y. Xin, C. You

\section{The University of Kansas, Lawrence, USA}

P. Baringer, A. Bean, C. Bruner, R.P. Kenny III, D. Majumder, M. Malek, W. Mcbrayer, M. Murray, S. Sanders, R. Stringer, Q. Wang

\section{Kansas State University, Manhattan, USA}

A. Ivanov, K. Kaadze, S. Khalil, M. Makouski, Y. Maravin, A. Mohammadi, L.K. Saini, N. Skhirtladze, S. Toda

\section{Lawrence Livermore National Laboratory, Livermore, USA}

D. Lange, F. Rebassoo, D. Wright 


\section{University of Maryland, College Park, USA}

C. Anelli, A. Baden, O. Baron, A. Belloni, B. Calvert, S.C. Eno, C. Ferraioli, J.A. Gomez, N.J. Hadley, S. Jabeen, R.G. Kellogg, T. Kolberg, J. Kunkle, Y. Lu, A.C. Mignerey, Y.H. Shin, A. Skuja, M.B. Tonjes, S.C. Tonwar

\section{Massachusetts Institute of Technology, Cambridge, USA}

A. Apyan, R. Barbieri, A. Baty, R. Bi, K. Bierwagen, S. Brandt, W. Busza, I.A. Cali, Z. Demiragli, L. Di Matteo, G. Gomez Ceballos, M. Goncharov, D. Gulhan, Y. Iiyama, G.M. Innocenti, M. Klute, D. Kovalskyi, Y.S. Lai, Y.-J. Lee, A. Levin, P.D. Luckey, A.C. Marini, C. Mcginn, C. Mironov, S. Narayanan, X. Niu, C. Paus, C. Roland, G. Roland, J. Salfeld-Nebgen, G.S.F. Stephans, K. Sumorok, M. Varma, D. Velicanu, J. Veverka, J. Wang, T.W. Wang, B. Wyslouch, M. Yang, V. Zhukova

\section{University of Minnesota, Minneapolis, USA}

A.C. Benvenuti, B. Dahmes, A. Evans, A. Finkel, A. Gude, P. Hansen, S. Kalafut, S.C. Kao, K. Klapoetke, Y. Kubota, Z. Lesko, J. Mans, S. Nourbakhsh, N. Ruckstuhl, R. Rusack, N. Tambe, J. Turkewitz

\section{University of Mississippi, Oxford, USA}

J.G. Acosta, S. Oliveros

\section{University of Nebraska-Lincoln, Lincoln, USA}

E. Avdeeva, R. Bartek, K. Bloom, S. Bose, D.R. Claes, A. Dominguez, C. Fangmeier, R. Gonzalez Suarez, R. Kamalieddin, D. Knowlton, I. Kravchenko, F. Meier, J. Monroy, F. Ratnikov, J.E. Siado, G.R. Snow

\section{State University of New York at Buffalo, Buffalo, USA}

M. Alyari, J. Dolen, J. George, A. Godshalk, C. Harrington, I. Iashvili, J. Kaisen, A. Kharchilava, A. Kumar, S. Rappoccio, B. Roozbahani

\section{Northeastern University, Boston, USA}

G. Alverson, E. Barberis, D. Baumgartel, M. Chasco, A. Hortiangtham, A. Massironi, D.M. Morse, D. Nash, T. Orimoto, R. Teixeira De Lima, D. Trocino, R.-J. Wang, D. Wood, J. Zhang

\section{Northwestern University, Evanston, USA}

S. Bhattacharya, K.A. Hahn, A. Kubik, J.F. Low, N. Mucia, N. Odell, B. Pollack, M. Schmitt, K. Sung, M. Trovato, M. Velasco

\section{University of Notre Dame, Notre Dame, USA}

N. Dev, M. Hildreth, C. Jessop, D.J. Karmgard, N. Kellams, K. Lannon, N. Marinelli, F. Meng, C. Mueller, Y. Musienko ${ }^{38}$, M. Planer, A. Reinsvold, R. Ruchti, G. Smith, S. Taroni, N. Valls, M. Wayne, M. Wolf, A. Woodard

\section{The Ohio State University, Columbus, USA}

L. Antonelli, J. Brinson, B. Bylsma, L.S. Durkin, S. Flowers, A. Hart, C. Hill, R. Hughes, W. Ji, T.Y. Ling, B. Liu, W. Luo, D. Puigh, M. Rodenburg, B.L. Winer, H.W. Wulsin 


\section{Princeton University, Princeton, USA}

O. Driga, P. Elmer, J. Hardenbrook, P. Hebda, S.A. Koay, P. Lujan, D. Marlow,

T. Medvedeva, M. Mooney, J. Olsen, C. Palmer, P. Piroué, D. Stickland, C. Tully, A. Zuranski

\section{University of Puerto Rico, Mayaguez, USA}

S. Malik

\section{Purdue University, West Lafayette, USA}

A. Barker, V.E. Barnes, D. Benedetti, D. Bortoletto, L. Gutay, M.K. Jha, M. Jones, A.W. Jung, K. Jung, A. Kumar, D.H. Miller, N. Neumeister, B.C. Radburn-Smith, X. Shi, I. Shipsey, D. Silvers, J. Sun, A. Svyatkovskiy, F. Wang, W. Xie, L. Xu

Purdue University Calumet, Hammond, USA

N. Parashar, J. Stupak

\section{Rice University, Houston, USA}

A. Adair, B. Akgun, Z. Chen, K.M. Ecklund, F.J.M. Geurts, M. Guilbaud, W. Li, B. Michlin, M. Northup, B.P. Padley, R. Redjimi, J. Roberts, J. Rorie, Z. Tu, J. Zabel

\section{University of Rochester, Rochester, USA}

B. Betchart, A. Bodek, P. de Barbaro, R. Demina, Y. Eshaq, T. Ferbel, M. Galanti, A. Garcia-Bellido, J. Han, O. Hindrichs, A. Khukhunaishvili, K.H. Lo, P. Tan, M. Verzetti

\section{Rutgers, The State University of New Jersey, Piscataway, USA}

J.P. Chou, E. Contreras-Campana, D. Ferencek, Y. Gershtein, E. Halkiadakis, M. Heindl, D. Hidas, E. Hughes, S. Kaplan, R. Kunnawalkam Elayavalli, A. Lath, K. Nash, H. Saka, S. Salur, S. Schnetzer, D. Sheffield, S. Somalwar, R. Stone, S. Thomas, P. Thomassen, M. Walker

\section{University of Tennessee, Knoxville, USA}

M. Foerster, G. Riley, K. Rose, S. Spanier, K. Thapa

\section{Texas A\&M University, College Station, USA}

O. Bouhali ${ }^{70}$, A. Castaneda Hernandez ${ }^{70}$, A. Celik, M. Dalchenko, M. De Mattia, A. Delgado, S. Dildick, R. Eusebi, J. Gilmore, T. Huang, T. Kamon ${ }^{71}$, V. Krutelyov, R. Mueller, I. Osipenkov, Y. Pakhotin, R. Patel, A. Perloff, A. Rose, A. Safonov, A. Tatarinov, K.A. Ulmer ${ }^{2}$

\section{Texas Tech University, Lubbock, USA}

N. Akchurin, C. Cowden, J. Damgov, C. Dragoiu, P.R. Dudero, J. Faulkner, S. Kunori, K. Lamichhane, S.W. Lee, T. Libeiro, S. Undleeb, I. Volobouev

\section{Vanderbilt University, Nashville, USA}

E. Appelt, A.G. Delannoy, S. Greene, A. Gurrola, R. Janjam, W. Johns, C. Maguire, Y. Mao, A. Melo, H. Ni, P. Sheldon, S. Tuo, J. Velkovska, Q. Xu 


\section{University of Virginia, Charlottesville, USA}

M.W. Arenton, B. Cox, B. Francis, J. Goodell, R. Hirosky, A. Ledovskoy, H. Li, C. Lin, C. Neu, T. Sinthuprasith, X. Sun, Y. Wang, E. Wolfe, J. Wood, F. Xia

\section{Wayne State University, Detroit, USA}

C. Clarke, R. Harr, P.E. Karchin, C. Kottachchi Kankanamge Don, P. Lamichhane, J. Sturdy

\section{University of Wisconsin - Madison, Madison, WI, USA}

D.A. Belknap, D. Carlsmith, M. Cepeda, S. Dasu, L. Dodd, S. Duric, B. Gomber, M. Grothe, M. Herndon, A. Hervé, P. Klabbers, A. Lanaro, A. Levine, K. Long, R. Loveless, A. Mohapatra, I. Ojalvo, T. Perry, G.A. Pierro, G. Polese, T. Ruggles, T. Sarangi, A. Savin, A. Sharma, N. Smith, W.H. Smith, D. Taylor, P. Verwilligen, N. Woods

$\dagger$ : Deceased

1: Also at Vienna University of Technology, Vienna, Austria

2: Also at CERN, European Organization for Nuclear Research, Geneva, Switzerland

3: Also at State Key Laboratory of Nuclear Physics and Technology, Peking University, Beijing, China

4: Also at Institut Pluridisciplinaire Hubert Curien, Université de Strasbourg, Université de Haute Alsace Mulhouse, CNRS/IN2P3, Strasbourg, France

5: Also at National Institute of Chemical Physics and Biophysics, Tallinn, Estonia

6: Also at Skobeltsyn Institute of Nuclear Physics, Lomonosov Moscow State University, Moscow, Russia

7: Also at Universidade Estadual de Campinas, Campinas, Brazil

8: Also at Centre National de la Recherche Scientifique (CNRS) - IN2P3, Paris, France

9: Also at Laboratoire Leprince-Ringuet, Ecole Polytechnique, IN2P3-CNRS, Palaiseau, France

10: Also at Joint Institute for Nuclear Research, Dubna, Russia

11: Also at British University in Egypt, Cairo, Egypt

12: Now at Suez University, Suez, Egypt

13: Also at Cairo University, Cairo, Egypt

14: Also at Fayoum University, El-Fayoum, Egypt

15: Also at Université de Haute Alsace, Mulhouse, France

16: Also at Tbilisi State University, Tbilisi, Georgia

17: Also at RWTH Aachen University, III. Physikalisches Institut A, Aachen, Germany

18: Also at University of Hamburg, Hamburg, Germany

19: Also at Brandenburg University of Technology, Cottbus, Germany

20: Also at Institute of Nuclear Research ATOMKI, Debrecen, Hungary

21: Also at Eötvös Loránd University, Budapest, Hungary

22: Also at University of Debrecen, Debrecen, Hungary

23: Also at Wigner Research Centre for Physics, Budapest, Hungary

24: Also at Indian Institute of Science Education and Research, Bhopal, India

25: Also at University of Visva-Bharati, Santiniketan, India

26: Now at King Abdulaziz University, Jeddah, Saudi Arabia

27: Also at University of Ruhuna, Matara, Sri Lanka

28: Also at Isfahan University of Technology, Isfahan, Iran

29: Also at University of Tehran, Department of Engineering Science, Tehran, Iran 
30: Also at Plasma Physics Research Center, Science and Research Branch, Islamic Azad University, Tehran, Iran

31: Also at Laboratori Nazionali di Legnaro dell'INFN, Legnaro, Italy

32: Also at Università degli Studi di Siena, Siena, Italy

33: Also at Purdue University, West Lafayette, USA

34: Also at International Islamic University of Malaysia, Kuala Lumpur, Malaysia

35: Also at Malaysian Nuclear Agency, MOSTI, Kajang, Malaysia

36: Also at Consejo Nacional de Ciencia y Tecnología, Mexico city, Mexico

37: Also at Warsaw University of Technology, Institute of Electronic Systems, Warsaw, Poland

38: Also at Institute for Nuclear Research, Moscow, Russia

39: Now at National Research Nuclear University 'Moscow Engineering Physics Institute' (MEPhI), Moscow, Russia

40: Also at St. Petersburg State Polytechnical University, St. Petersburg, Russia

41: Also at California Institute of Technology, Pasadena, USA

42: Also at Faculty of Physics, University of Belgrade, Belgrade, Serbia

43: Also at INFN Sezione di Roma; Università di Roma, Roma, Italy

44: Also at National Technical University of Athens, Athens, Greece

45: Also at Scuola Normale e Sezione dell'INFN, Pisa, Italy

46: Also at National and Kapodistrian University of Athens, Athens, Greece

47: Also at Institute for Theoretical and Experimental Physics, Moscow, Russia

48: Also at Albert Einstein Center for Fundamental Physics, Bern, Switzerland

49: Also at Adiyaman University, Adiyaman, Turkey

50: Also at Mersin University, Mersin, Turkey

51: Also at Cag University, Mersin, Turkey

52: Also at Piri Reis University, Istanbul, Turkey

53: Also at Gaziosmanpasa University, Tokat, Turkey

54: Also at Ozyegin University, Istanbul, Turkey

55: Also at Izmir Institute of Technology, Izmir, Turkey

56: Also at Marmara University, Istanbul, Turkey

57: Also at Kafkas University, Kars, Turkey

58: Also at Istanbul Bilgi University, Istanbul, Turkey

59: Also at Yildiz Technical University, Istanbul, Turkey

60: Also at Hacettepe University, Ankara, Turkey

61: Also at Rutherford Appleton Laboratory, Didcot, UK

62: Also at School of Physics and Astronomy, University of Southampton, Southampton, UK

63: Also at Instituto de Astrofísica de Canarias, La Laguna, Spain

64: Also at Utah Valley University, Orem, USA

65: Also at University of Belgrade, Faculty of Physics and Vinca Institute of Nuclear Sciences, Belgrade, Serbia

66: Also at Facoltà Ingegneria, Università di Roma, Roma, Italy

67: Also at Argonne National Laboratory, Argonne, USA

68: Also at Erzincan University, Erzincan, Turkey

69: Also at Mimar Sinan University, Istanbul, Istanbul, Turkey

70: Also at Texas A\&M University at Qatar, Doha, Qatar

71: Also at Kyungpook National University, Daegu, Korea 